\title{
Conservative treatment of caustic oesophageal injuries in children: 15 years of experience in a tertiary care paediatric centre
}

\author{
Jonas Povilavičius ${ }^{1}$, Narimantas Evaldas Samalavičius ${ }^{2,3}$, Gilvydas Verkauskass ${ }^{1}, K_{\text {Kętutis Trainavičius }}{ }^{1}$, \\ Milda Povilavičiené ${ }^{4}$ \\ ${ }^{1}$ Department of Paediatric Surgery, Children's Hospital, Affiliate of Vilnius University Hospital Santaros Klinikos, Vilnius, Lithuania \\ ${ }^{2}$ Department of Surgery, Klaipeda University Hospital, Klaipeda, Lithuania \\ ${ }^{3}$ Clinic of Internal, Family Medicine and Oncology, Faculty of Medicine, Vilnius University, Vilnius, Lithuania \\ ${ }^{4}$ Department of Surgery, Ukmergès Hospital, Ukmergė, Lithuania
}

Gastroenterology Rev 2019; 14 (4): 286-291

DOI: https://doi.org/10.5114/pg.2019.90255

Key words: caustic injury, conservative treatment, endoscopic dilatation.

Address for correspondence: DSurg Jonas Povilavičius, Department of Paediatric Surgery, Children's Hospital, Affiliate of Vilnius University Hospital Santaros Klinikos, Vilnius, Lithuania, phone: +37 061559844, e-mail: jonas.povilavicius@gmail.com

\begin{abstract}
Introduction: Endoscopic dilatation is good choice of conservative treatment for caustic digestive tract injuries in children. Aim: To set up a strategy of management of caustic digestive tract injury based on our experience and literature review.

Material and methods: We retrospectively analysed medical records of 34 paediatric patients who were admitted to the Centre of Paediatric Surgery of the Children's Hospital between 2000 and 2017. Age at presentation, gender, anatomic location, circumstances and distribution of injury, early and late complications, clinical signs, and the first aid were analysed. Upper gastrointestinal (GI) endoscopy was performed within 12-24 h after ingestion in all cases. The Zargar classification system was used to grade the severity of the injury. Fisher's exact test was used for statistical analysis, with $p<0.05$ considered as the limit of statistical significance.

Results: The upper Gl endoscopy revealed caustic injuries in $5(15 \%)$ and $8(23 \%)$ patients were classified as grade Ila and $\mathrm{IIb}$, respectively. Oesophageal and ventricle caustic injuries in $3(9 \%)$ and $2(6 \%)$ patients were classified as grade IIIa and IIIb, respectively. Thirteen patients with grade IIa and IIIb injuries suffered permanent damage and required repeated dilatation. All patients underwent stricture treatment using late or early endoscopic dilatation of the oesophagus. An average of 15 dilatation procedures were required to achieve a satisfactory lumen.

Conclusions: Our experience of 34 children revealed that endoscopic dilatation may be required as a primary treatment for oesophageal strictures.
\end{abstract}

\section{Introduction}

Accidental caustic ingestion and consequential oesophageal strictures remain major paediatric health problems worldwide [1]. Commonly available household products, such as dishwashing liquids, window cleaning agents, drain cleaners, vinegar essence, and potassium permanganate, are highly corrosive agents if accidentally ingested. The increased availability of such products has led to an increased number of accidental caustic ingestions in the paediatric population [2]. Most of the ingestion injuries worldwide occur in children, mainly due to accidental ingestion [3]. Proper labelling, antidote instructions, concentration restrictions, and child-resistant packaging can significantly reduce the number of such cases.

The caustic agents in household products may be acidic or alkaline, causing different reactions and sequelae. The concentrations of the ingested material and the amount swallowed determine the severity of the injury. The clinical spectrum of paediatric caustic ingestion can vary from no apparent injury to potentially fatal and severe complications like oesophageal perforations, strictures requiring multiple dilatations. Although several treatment strategies have been described and guidelines were issued by the European 
Society of Gastrointestinal Endoscopy (ESGE) and the European Society for Paediatric Gastroenterology Hepatology and Nutrition (ESPGHAN), controversy remains as to the timing in treatment protocols, choice of dilatation technique, and the results in the acute or chronic phase of caustic ingestion [4-9].

\section{Aim}

To set up a strategy of management of caustic digestive tract injury based on our experience and literature review.

\section{Material and methods}

We retrospectively analysed medical records of 34 paediatric patients who were admitted to the Centre of Paediatric Surgery of the Children's Hospital between 2000 and 2017.

The following patient parameters were analysed: age at presentation, gender, nature and contact time of the ingested caustic substance, circumstances of injury (accidental or intentional), anatomic location and distribution of injury, early and late complications, general condition, clinical signs, and the first aid when they were admitted to the hospital. Antacid therapy (weightbased omeprazole or pantoprazole and ranitidine daily), antibiotics (co-amoxiclav), laryngoscopy, and upper gastrointestinal (Gl) endoscopy were performed within 12-24 h of ingestion in all cases. The Zargar classification system was used to grade the severity of the injury (Table I).

Twenty-seven boys and seven girls, median age 21.5 months at presentation, were analysed with an average follow-up period of 5 years. All cases were accidental ingestions. No mortality occurred in the series. Twenty-four (71\%) incidents of ingestion had occurred in a home, $9(27 \%)$ in a garage, and one in a garden. Alkali comprised 23 (67\%) cases, acid 8 (24\%) cases, chlorine bleach 1 (3\%) case, and glue and unknown liquid $2(6 \%)$ cases. The most frequently ingested alkalis were dishwashing powder, disinfectants, caustic soda used for cleaning ovens, and degreasers. Half of the children had sipped liquids straight from a container, cup, or bottle, whereas 24 (48\%) children had ingested the caustic substances in powdered or granulated forms. Six (12\%) children had picked up cups or bottles, which were used for keeping decanted caustic solutions (Table II).

Before arriving at the hospital, the main clinical symptoms were drooling (55\%), vomiting (23\%), painful swallowing (17\%), and dyspnoea (17\%). Haematemesis (3\%) and dysphonia (11\%) were also noted. Before arriving at the hospital, 16 (47\%) children received first aid in the form of gastric lavage by emergency care specialists. All patients underwent upper GI endosco- py within 12-24 h of ingestion. After establishing the diagnosis, the treatment protocol with an appropriate dosage of a proton-pump inhibitor, analgesics, and co-amoxiclav was commenced. Fisher's exact test was used for statistical analysis, with $p<0.05$ set as the limit of statistical significance.

\section{Ethics}

Ethical considerations and approval were in accordance with the World Medical Association Declaration of Helsinki. The Institutional Review Board of the Children's Hospital approved all aspects of this study. Approval was provided for research involving the use of data records originally collected for non-research purposes.

Table I. Zargar classification and its corresponding endoscopic description

\begin{tabular}{|c|c|}
\hline Grade & Characteristics \\
\hline 0 & Normal mucosa \\
\hline । & Oedema and erythema of the mucosa \\
\hline Ila & $\begin{array}{c}\text { Haemorrhage, erosions, blisters, superficial } \\
\text { ulcers }\end{array}$ \\
\hline$\| \mathrm{b}$ & Circumferential lesions \\
\hline IIIa & Focal deep grey or brownish-black ulcers \\
\hline IIIb & Extensive deep grey or brownish-black ulcers \\
\hline IV & Perforation \\
\hline
\end{tabular}

Table II. Patient characteristics, caustic agents, and mechanism of consumption

\begin{tabular}{lc} 
Parameter & Value \\
\hline Total patients & 34 (27 boys, 7 girls $)$ \\
\hline Median age (range) & $21.5(1-48$ months $)$ \\
\hline Ingested substance: & $67 \%$ \\
\hline Alkali & $24 \%$ \\
\hline Acid & $3 \%$ \\
\hline Chlorinated bleach & $3 \%$ \\
\hline Glue & $3 \%$ \\
\hline Unknown liquid & $9 \%$ \\
\hline Form of agent: & $91 \%$ \\
\hline Granules & $100 \%$ \\
\hline Liquid & 0 \\
\hline Circumstance of ingestion: & \\
\hline Accidental & \\
\hline Deliberate/inflicted harm & \\
\hline
\end{tabular}


Table III. Clinical outcomes of caustic ingestion

\begin{tabular}{lc} 
Mortality & Percent \\
\hline Grade 0 & 2 \\
\hline Grade I & 14 \\
\hline Grade Ila & 5 \\
\hline Grade IIb & 8 \\
\hline Grade IIIa & 3 \\
\hline Grade IIIb & 2
\end{tabular}

\section{Results}

The upper Gl endoscopy revealed no mucosal changes in two (6\%) patients and mild mucosal changes classified as grade 1 injury in the oesophagus in $14(41 \%)$ patients. Caustic injuries in 5 (15\%) and 8 (23\%) patients were classified as grade IIa and IIb, respectively. Oesophageal and gastric caustic injuries in 3 (9\%) and $2(6 \%)$ patients were classified as grade IIIa and IIIb, respectively (Table III). Generally, 18 (53\%) patients had Ila-IIIb caustic injuries, of whom 15 (83\%) were injured with alkali and three (17\%) with acid. Although there was no statistical significant correlation between these groups ( $p<0.35$ ), we found that 13 patients with grade IIa and IIIb injuries suffered permanent damage from alkali and required repeated dilatation. All patients underwent stricture treatment using late or early endoscopic dilatation of the oesophagus. An average of 15 dilatation procedures were required to achieve a satisfactory lumen. One child showed evidence of oesophageal changes without clinically detected oropharyngeal lesions. Eight (24\%) patients had laryngeal trauma, but only two of them required temporary intubation in the intensive care unit. No long-term laryngeal sequelae were observed in any child. All patients were hospitalised in the paediatric surgery unit and followed until the symptoms of dysphagia disappeared.

\section{Discussion}

Ingestion of caustic substances is a serious problem with consequences for the entire family, possibly leading to family break-ups, abandonment, and job losses. The biggest risk group for accidental caustic ingestion comprises children below 5 years old, and, as in our group, the incidence peaks at approximately 2 years of age, when children develop skills of localisation but have poor discrimination between harmless and harmful substances.

The extent and severity of the caustic injury to the oesophagus depends on the interactions of four factors: the corrosiveness of the ingested substance, its quanti- ty and concentration, and the duration of contact time. Crystals or solid particles may adhere to the oral mucosa, making swallowing difficult and diminishing the injury to the oesophagus but potentially increasing the damage to the upper airway and pharynx. Liquids can be easily swallowed and are most likely to damage the oesophagus and stomach, and the extent of injury is directly correlated with mortality and late sequelae [5]. Different household substances ranging from alkalotic bases with $\mathrm{pH}$ of up to 12 to acidic substances with $\mathrm{pH}$ as low as 2 as well as bleaching substances with $\mathrm{pH}$ of approximately 7 can cause caustic injuries. High concentrations of alkali are found in lye-based (sodium hydroxide/potassium hydroxide) drain cleaners, ammonia, and dishwashing soap. We think that every detail of the accident is very important to predict the severity of caustic injury.

The management of caustic injuries depends on accurate diagnosis. Because ingestion is often unwitnessed, careful history of the brand name, type, and amount of the ingested substance can be difficult to find out. Complaints of dyspnoea, dysphagia, excessive salivation, haematemesis, or hoarseness suggest severe injury [6]. Symptoms such as substernal chest pain, abdominal pain, and rigidity suggest profound injury and perforation of the oesophagus or stomach. Only stridor and drooling are considered $100 \%$ specific for significant oesophageal injury [7], but no single symptom or symptom cluster can predict the degree of oesophageal damage [8]. We found out that drooling and vomiting were the most important symptoms to suspect caustic injury.

Deep injury occurs when crystalline drain cleaners adhere to natural points of constriction of the oesophagus. The cricopharyngeus muscle, the area of compression by the aorta and left main bronchus, and the lower oesophageal sphincter are anatomical constrictions affected by caustic injuries. The development of oesophageal strictures depends on the depth and circumferential nature of the caustic injury. For example, burns caused by alkaline substances show liquefaction necrosis that may extend to the muscle layer. On the other hand, acidic substances cause coagulative necrosis only at the mucosal level when the concentration is low and the contact time is short. Alkali ingestion, especially lye, is associated with more severe oesophageal lesions; severe gastric lesions are likely to occur in acidic ingestion. Stricture development has been associated with both acidic and alkali ingestion [9]. In our experience, lye was the main cause of oesophageal strictures. Large quantities of alkaline and acidic substances can be ingested because of their innocuous taste, and the amount of caustic substance ingested affects the injury 
grade. In addition, alkali absorption leads to thrombosis in blood vessels, impeding blood flow to the already damaged tissue [10].

Oesophageal injury begins within minutes and may persist for hours. Forty-eight hours after ingestion, fibroblast proliferation and collagen synthesis begin and the oesophageal wall weakens, with weakness peaking at 1-3 weeks. The healing phase results in fibrosis and stricturing after approximately 3 weeks. The wound-healing events involving synthesis and deposition of proteins and wound contraction predominate 4-5 days after injury [11]. Moreover, compared with alkali ingestion, strong acid ingestion is associated with a higher incidence of systemic complications, such as renal failure, liver dysfunction, disseminated intravascular coagulation, and haemolysis [12]. There were no systemic complications detected in our group.

Lateral neck and chest X-rays should be routinely performed if perforation is suspected. Barium swallows are of little use in the acute phase because they delay upper GI endoscopy and do not reveal the degree of mucosal injuries; therefore, they were not used in our patients. Upper Gl endoscopy is crucial for further management. Grading of Gl endoscopy findings based on the severity of the injury can predict long-term outcome. Using careful upper GI endoscopic evaluation, unnecessary therapeutic interventions can be avoided in many cases. We hospitalised all patients with suspected caustic injury independent of symptoms. Upper $\mathrm{Gl}$ endoscopy was performed $12-24 \mathrm{~h}$ after ingestion, and patients were discharged home if there were no endoscopic findings of caustic injury.

Current contraindications for esophagoscopy are evident perforation, supraglottic or epiglottic burns with oedema, and third-degree burns in the hypopharynx [13]. However, when upper Gl endoscopy under anaesthesia is performed by a qualified team, the risk of perforation is low and the procedure can be completed even in the presence of second- or third-degree burns [14]. Hence, we did not find contraindications for upper $\mathrm{Gl}$ endoscopy in our group of patients. A retrospective review of 273 patients in 2008 revealed that endoscopic grading helped to predict outcomes, particularly in patients with grade III injuries [15]. Thus, the timing of the first upper $\mathrm{Gl}$ endoscopy is crucial.

The grade of the injury determined on careful endoscopic assessment and physical examination indicates the degree of urgency for surgical intervention, the development of subsequent complications, and eventual mortality [16]. Therefore, timing of the Gl endoscopy should be within the first $24 \mathrm{~h}$ [9]. We suggest a 12-24hour interval because early upper $\mathrm{Gl}$ endoscopy within $12 \mathrm{~h}$ may not accurately reveal the depth of an injury.
After 12-24 h, the effects of the injury will have established, and the severity can be appropriately graded. Upper Gl endoscopy performed after $72 \mathrm{~h}$ increases the risk of iatrogenic perforation because of potential structural weakness in the oesophageal wall. To predict the outcome in our series, the Zargar grading system was used, in which grade 1 indicates superficial injuries, grade 2 indicates transmucosal injuries, and grade 3 indicates transmural injuries. Some authors have recommended nasogastric intubation during the first upper Gl endoscopy to ensure feeding and to prevent oesophageal stricturing. However, we favour sufficient analgesics and the permission of oral feeds for the better comfort of not having to use a feeding tube. In addition, nasogastric intubation may cause fungal esophagitis after caustic injury.

Bleaches cause a different set of caustic injuries. Household bleaches that are weak alkalis cause ulcerations that usually do not result in stricture. In our series, only one child had sustained caustic injury due to bleach ingestion, with no permanent sequela or stricture noted after treatment.

In the literature there is some evidence showing that antibiotics markedly reduce the incidence of stricture formation by decreasing bacterial counts in the necrotic tissue, thus reducing superinfection of the granulation tissue, especially if steroids are used together. In our cases, co-amoxiclav was routinely administered for 10 days, starting from grade Ila, possibly contributing to the results.

Induced emesis and gastric lavage are contraindicated to avoid further exposure of the oesophagus to the caustic agent. Administration of neutralising agents is also contraindicated because they cause exothermic reactions, thereby further injuring the tissue. Our experience had shown that 16 (47\%) children received first aid in the form of gastric lavage before arriving to our hospital.

The use of corticosteroids for treating caustic injuries of the digestive tract remains controversial. Multiple trials and reviews have shown little or no measurable benefit of various steroid doses in terms of their ability to reduce the rate of stricture formation [17]. In addition, evidence has shown that steroids are most beneficial in preventing stricture formation in grade $\mathrm{Ilb}$ injuries, and there is no evidence of benefit of the use of corticosteroids in other grades of esophagitis (I, Ila, III) [17]. Steroid administration is justified only when upper airway oedema and laryngeal injury are suspected [18]. We found no indication to use steroids in our cases. However, high doses of steroids are included in our treatment protocol for grade IIb caustic injuries of the digestive tract, as recommended by guidelines [9]. 
Secondary damage due to gastroesophageal reflux may highly implicate stricture formation. The routine administration of anti-reflux therapy is recommended for the prevention of secondary reflux-associated oesophageal injury. The efficacy of this has not been proven, but a small study performed in 2013 showed endoscopic healing after omeprazole infusion [19]. Our patients were administered proton-pump inhibitors, such as omeprazole and esomeprazole, and $\mathrm{H} 2$ antagonists, such as ranitidine until discharge.

Most strictures occur in the oesophagus [20]. In our series, $26 \%$ of grade IIa and III cases developed oesophageal strictures requiring further treatment, although some studies have shown that following a grade IIb or III oesophageal burn, stricture incidence may be from $71 \%$ to $100 \%$ [21], respectively.

Coexistent gastric damage can extend from simple hyperaemia/erosions to diffuse transmural necrosis. In our series the gastric injury rate was $26 \%$.

Although the treatment of caustic oesophageal strictures is challenging, time consuming, and in many cases unsatisfactory [22], dilatation remains the primary choice of treatment [23]. The success rate, unless contraindicated, is $60-80 \%$ in most centres. Timely evaluation and dilatation of the stricture are crucial for achieving a favourable outcome [23]. In our series, all patients with oesophagus strictures were successfully treated by this approach.

Repeated antegrade or retrograde dilatations is a widely accepted treatment strategy of oesophageal strictures after caustic ingestion [4]. In our series, patients with strictures underwent first dilatation 2 weeks after the injury; mainly antegrade dilatation was performed using Savary-Gilliard dilators over a guidewire. The optimal frequency and time of dilatation procedures is largely individualised. The time interval between procedures is based on the effects of previous dilatations and symptomatology [24]. Our patients underwent dilatation once or twice every week until there was no resistance to the dilatator, depending on the age. Procedures in all patients were performed under general endotracheal anaesthesia. The patients were discharged home when the symptoms of dysphagia disappeared. Although endoscopic dilatation is still considered the first line of treatment for preserving the native oesophagus, multiple sessions over a long period of time (up to 2-3 years) may be required [25]. All dilatation procedures can be performed using a balloon or bougie (usually Savary), but no procedure shows a clear advantage [26]. Savary bougies are considered more reliable than balloon dilators in consolidated and fibrotic strictures, such as old caustic stenosis, or in long and tortuous strictures [27, 28], and may offer the operator the advantage of feeling that the dilatation is occurring under his hands [29]. Although oesophageal dilatation can be safe and effective when performed by experienced hands, several problems like perforations and even death could occur in patients with severe strictures. In our series, such complications were never experienced; this could be attributed to the considerable experience of the staff in using Savary-Gilliard dilators over a guidewire under endoscopic control.

Oesophageal neoplasms (both adenocarcinoma and squamous cell carcinoma) may develop as late complications of caustic injuries at a rate 1000-3000 times higher than that expected in patients of a similar age [30]. Future follow-up of these patients should be continued.

The drawbacks of our study we attribute to small sample size, retrospective design, and the single-centre experience.

\section{Conclusions}

Endoscopic dilatation may be required as a primary treatment for oesophageal strictures. The frequency and timing of dilatation procedures are individualised and based on symptomatology and previous procedures. All suspected cases of ingestions should be evaluated by upper Gl endoscopy within 12-24 h of the ingestion event for accurate diagnosis with recommend administration of broad spectrum antibiotics, antacid therapy, and conservative treatment with Savary-Gilliard dilators over a guidewire under endoscopic control for the treatment and prevention of oesophageal strictures.

\section{Conflict of interest}

The authors declare no conflict of interest.

\section{References}

1. Riffat F, Cheng A. Pediatric caustic ingestion: 50 consecutive mases and a review of the literature. Dis Esophagus 2009; 22: 89-94.

2. Litovitz TL, Klein-Schwartz W, Rodgers GC Jr, et al. 2001 Annual report of American Association of Poison Control Centers Toxic Exposure Surveillance system. Am J Emerg Med 2002; 20: 391-452.

3. Watson WA, Litovitz TL, Rodgers GC, et al. 2004 Annual report of the American Association of Poison Control Centers Toxic Exposure Surveillance System. Am J Emerg Med 2005; 23: 589-666.

4. Ashcraft KW. The esophagus. In: Pediatric Surgery. 3rd edn. Ashcraft KW (ed). WB Saunders, Philadelphia 2000; 325-47.

5. Salzman M, O'Malley RN. Updates on the evaluation and management of caustic exposures. Emerg Med Clin North Am 2007; 25: 459-76.

6. Rigo GP, Camellini L, Azzolini F, et al. What is the utility of selected clinical and endoscopic parameters in predicting the 
risk of death after caustic ingestion? Endoscopy 2002; 34: 304-10.

7. Havanond C, Havanond P. Initial signs and symptoms as prognostic indicators of severe gastrointestinal tract injury due to corrosive ingestion. J Emerg Med 2007; 33: 349-53.

8. Gupta SK, Croffie JM, Fitzgerald JF. Is esophagogastroduodenoscopy necessary in all caustic ingestions? J Pediatr Gastroenterol Nutr 2001; 32: 50-3.

9. Thomson M, Tringali A, Dumonceau JM, et al. Pediatric gastrointestinal endoscopy: European Society for Paediatric Gastroenterology Hepatology and Nutrition (ESPGHAN) and European Society of Gastrointestinal Endoscopy (ESGE) Guidelines. J Pediatr Gastroenterol Nutr 2017; 49: 83-91.

10. Mamede RC, de Mello Filho FV. Ingestion of caustic substances and its complications. Sao Paulo Med J 2001; 119: 10-5.

11. Monaco JL, Lawrence WT. Acute wound healing: an overview. Clin Plast Surg 2003; 30: 1-12.

12. Poley JW, Steyerberg EW, Kuipers EJ, et al. Ingestion of acid and alkaline agents: outcome and prognostic value of early upper endoscopy. Gastrointest Endosc 2004; 60: 372-7.

13. Ramasamy K, Gumaste VV. Corrosive ingestion in adults. J Clin Gastroenterol 2003; 37: 119-24.

14. Contini S, Tesfaye M, Picone P, et al. Corrosive esophageal injuries in children. A shortlived experience in Sierra Leone. Int J Pediatr Otorhinolaryngol 2007; 71: 1597-604.

15. Cheng $\mathrm{HT}$, Cheng $\mathrm{CL}$, Lin $\mathrm{CH}$, et al. Caustic ingestion in adults: the role of endoscopic classification in predicting outcome. BMC Gastroenterol 2008; 8: 31.

16. Poley JW, Steyerberg EW, Kuipers EJ, et al. Ingestion of acid and alkaline agents: outcome and prognostic value of early upper endoscopy. Gastrointest Endosc 2004; 60: 372-7.

17. Poley JW, Steyerberg EW, Kuipers EJ, et al. Ingestion of acid and alkailen agents: outcome and prognostic value of early endoscopy. Gastrointest Endosc 2004; 60: 372-7.

18. Huang Y, Ni Y, Lai H, et al. Corrosive esophagitis in children. Pediatr Surg Int 2004; 20: 207-10.

19. Rathnaswami A, Ashwin R. Corrosive injury of the upper gastrointestinal tract: a review. Arch Clin Gastroenterol 2016; 2: 56-62.

20. Lebeau R, Coulibaly A, Kountele Gona S, et al. Isolated gastric outlet obstruction due to corrosive ingestion. J Visc Surg 2011; 148: 59-63.

21. Kay M, Wyllie R. Caustic ingestions in children. Curr Opin Pediatr 2009; 21: 651-4.

22. Repici A, Conio M, De Angelis C, et al. Temporary placement of an expandable polyester silicone-covered stent for treatment of refractory benign esophageal strictures. Gastrointest Endosc 2004; 60: 513-9.

23. Doğan Y, Erkan T, Cokuğraş FC, et al. Caustic gastroesophageal lesions in childhood: an analysis of 473 cases. Clin Pediatr (Phila) 2006; 45: 435-8.

24. De Jong AL, Macdonald R, Ein S, et al. Corrosive esophagitis in children: a 30-year review. Int J Pediatr Otorhinolaryngol 2001; 57: 203-11.

25. Poddar U, Thapa BR. Benign esophageal strictures in infants and children: results of Savary-Gilliard bougie dilation in 107 Indian children. Gastrointest Endosc 2001; 54: 480-4.
26. Siersema PD, de Wijkerslooth LR. Dilation of refractory benign esophageal strictures. Gastrointest Endosc 2009; 70: 1000-12.

27. Dall'Oglio L, De Angelis P. Commentary on "Esophageal endoscopic dilations". J Pediatr Gastroenterol Nutr 2012; 54: 716-7.

28. Lakhdar-Idrissi M, Khabbache K, Hida M. Esophageal endoscopic dilations. J Pediatr Gastroenterol Nutr 2012; 54: 744-7.

29. Shehata SM, Enaba ME. Endoscopic dilatation for benign oesophageal strictures in infants and toddlers: experience of an expectant protocol from North African tertiary centre. Afr J Paediatr Surg 2012; 9: 187-92.

30. Park KS. Evaluation and management of caustic injuries from ingestion of Acid or alkaline substances. Clin Endosc 2014; 47: 301-7.

Received: 10.10 .2018

Accepted: 28.03.2019 